\title{
BERKELEY Y BENACERRAF \\ LA ARITMÉTICA ES SÓLO UN SISTEMA DE SIGNOS*
}

JOSE A. ROBLES

Instituto de Investigaciones Filosoficas

UNAM

\section{Propósito}

En este escrito presento las tesis de Berkeley acerca de la aritmética (y el álgebra) que figuran de manera central, pero no exclusiva en sus Comentarios filosoficos ( $C F$ de ahora en adelante) y muestro la gran similitud que las mismas tienen con la propuesta que sobre estos temas formulara en nuestro siglo Paul Benacerraf. ${ }^{1}$ De manera menos detallada formulo, también, otra tesis de similitud, tomando nuevamente propuestas de Ber-

* Leí una primera versión de este escrito en el V Congreso Nacional de Filosofía (Xalapa, Ver., oct. 1989). En el capítulo 2 de mi libro Las ideas matemáticas de George Berkeley (en prensa), entre otras cosas, presenté la propuesta a favor de la relación entre las tesis de Berkeley y de Hartry Field acerca del papel puramente auxiliar de las matemáticas en la ciencia; de la relación entre Berkeley y Benacerraf me hizo consciente la lectura de la disertación doctoral de Carlos Alfonso Ávila, quien analiza algunas de las ideas de Benacerraf.

Leí una versión ampliada en el Simposio de Filosofía de las matemáticas: La existencia y el conocimiento de las entidades matemáticas, que se llevó a cabo en el Instituto de Investigaciones Filosóficas (UNAM), del 4 al 6 de septiembre de 1990. La presente versión recoge observaciones que me hicieron algunos de los participantes en esta reunión: Keith Hossack, León Olivé, Pedro Ramos y Francisco Rodríguez Consuegra. Aquí les agradezco la oportunidad que me dieron de precisar más las ideas contenidas en este texto.

1 Cfr. [2] en la Bibliografía 
keley pero relacionándolas, ahora, con las de Hartry Field. ${ }^{2}$ Lo que esto muestra es la visión por demás de vanguardia de Berkeley sobre asuntos matemáticos. Las tesis mejores y más fundadas de Berkeley sobre temas matemáticos se encuentran articuladas en su importante publicacion de 1734 The Analyst; las mismas son, sin embargo, críticas de los endebles (o inexistentes) fundamentos del cálculo naciente y este tema lo he tocado de manera más, amplia en otro lugar, ${ }^{3}$ por lo que aquí no hablaré de él.

Algo que no pretendo hacer en este trabajo es considerar las propuestas berkeleyanas dentro del contexto más amplio de su filosofía general, pues esto me obligaría a presentar un estudio detallado de su doctrina de los signos, de los problemas que se presentarían al tomar en serio su propuesta central, esse est percipi, en un intento de proponer una fundamentación de la aritmética elemental y algunas cuestiones más que rebasan con mucho los límites de este trabajo.

Haciendo de lado el que sea posible o no ofrecer una fundamentación de la aritmética elemental dentro del contexto de la filosofía de Berkeley, lo que me interesa subrayar, como lo sugerí al comenzar este escrito, es la lucidez que tuvo Berkeley al proponer tesis que aún tienen vigencia, formuladas hace unos 300 años por el pensador irlandés, en una época en la que se han logrado muy notables avances, técnicos tanto en lógica como en matemáticas, así como con respecto a nuestra comprensión de los sistemas numéricos, con respecto a todo lo cual había bastante oscuridad y confusión en el siglo XVIII.

2 Hartry Field —en [10] — presenta un interesante estudio del nominalismo en matemáticas. Fue a partir de la lectura de partes de este libro que obtuve la idea de leer a Berkeley de la manera como lo hago, más adelante, en el texto principal.

3 En [12], cap. 4. 


\section{Introducción: las tesis de Berkeley sobre la aritmética}

Berkeley se interesó en la matemática desde el principio mismo de sus meditaciones filosóficas, en sus Comentarios filosóficos (redactados en 1707-1708), en su De Ludo Algebraico y en su Miscellanea Mathematica (1707; publicados, en un sólo volumen, para tener derecho a optar por una plaza de miembro - fellow - del Trinity College) y en su De infinitos (leído en 1709). Pero hay que decir algo más acerca del tipo de este interés. En varios pasajes a lo largo de sus obras, a Berkeley mucho le preocupa la búsqueda del tipo de conocimiento que sea más útil para el beneficio de la humanidad. Respecto a las matemáticas piensa que la mayor parte de ellas son inútiles, al carecer de cualquier objeto de estudio. Algunos de sus comentarios son:

Cómo pueden llevarse tan bien puesto que en ellas (me refiero a las matemáticas) hay tantas contradictoriæ argutiæ v. Barrow Lect: $(C F 334)^{4}$

4 En esta anotación Berkeley se refiere al distinguido matemático inglés Isaac Barrow (1630-1677), quien fuera maestro de Newton en Cambridge, primer ocupante, en 1663 , de la cátedra Lucasiana de geometría en esa universidad. En 1669 (calendario juliano) renuncia a la cátedra a favor de Newton.

Barrow hizo una edición completa de los Elementos de Euclides y primero la publicó en latín, luego en inglés en 1660. En 1683 se hizo una publicación póstuma de las cátedras que dictó en los años 1664-1666, con el título Lectiones Mathematica. Por otra parte, durante su vida, publicó sus Lectiones Opticae et Geometrica en 1669. (Cfr. [1], pp. 309-310.) Luce _en [3], p. 385_, señala que Berkeley considera aquí Lect. Math., V.

Ahora quiero añadir otro de los argumentos de Berkeley a favor de su interpretación nominalista de la aritmética. El argumento está contenido en una carta a Molyneux (Samuel, el hijo de William) del 8 de diciembre de 1709. Por esta época Berkeley ya había publicado su Nueva teoría de la visión y sus Principios estaban casi listos para publicarse. El argumento sigue la línea de sus pensamientos en los Comentarios. Berkeley le dice a Molyneux que:

Muy bien podemos y, en mi opinión, lo hacemos con frecuencia, razonar sin Ideas pero sólo que las palabras que se usan, al usarse en su mayor parte 
¿Dónde se encuentra la necesidad de certeza en tales fruslerías? lo que hace que en ellas se les estime tanto es que se piensa que no somos capaces de obtenerla en otro lugar. Pero podemos en Ética y en Metafísica. (CF 336)

La arenga de Locke acerca de los Discursos coherentes, metódicos que no valen nada, aplicada a los Matemáticos. ( $C F$ 574)

En los Principios dijo:

Por tanto, las teorías en aritmética, si se abstraen de los nombres y las figuras, así como de cualquier uso y aplicación, tanto como de las cosas particulares enumeradas, puede suponerse que no tienen nada en absoluto como su objeto. De aquí podemos ver cuán completamente está subordinada la ciencia de los números a la práctica y cuán vacía y trivial se hace cuando se la considera como un asunto de mera especulación. ( $P C H \mathrm{I}, 120)$

En la siguiente sección Berkeley va en contra de quienes

gastan su tiempo en teoremas y problemas aritméticos que no tienen uso alguno. (PCH I, 121)

Una vez con los pasajes anteriores a la mano claramente podemos ver que Berkeley no estaba dispuesto, en manera alguna, a desperdiciar su tiempo en especulaciones matemáticas que

como las Letras en Álgebra, que aun cuando denotan Cantidades particulares, Empero cada paso no nos las sugiere a nuestros Pensamientos y, a pesar de eso Nosotros podemos razonar o realizar Operaciones totalmente acerca de ellas. De los números Nosotros no podemos forjar ninguna Noción más allá de cierto Grado y, sin emibargo, podemos razonar tan bien acerca de Mil como acerca de cinco, la Verdad de esto es que los Números no son nada sino Nombres. (En [9], p. 25)

El argumento no lo inaugura Berkeley, sin embargo; Descartes ya había señalado algo similar en las Meditations y Locke mismo usa un argumento parecido para mostrar cuán útiles son los signos en aritmética. Lo que es nuevo en el argumento de Berkeley es su conclusión de la no existencia de números a partir de la imposibilidad de representarlos. Una conclusión más débil, pero mejor fundada, sería decir que los números como entidades son inútiles y esto dejaría abierto el problema de su existencia o no existencia. 
no tuviesen ningún uso o aplicación inmediata. Pero también es claro que mantener una posición similar a la de Berkeley con respecto a las matemáticas es impedirles su crecimiento y la posibilidad de que, por esta misma razón, puedan ofrecernos algunas nuevas propuestas que podrían convertirse (en un tiempo largo o no tanto) en útiles en el sentido práctico que quería Berkeley.

De una manera perfectamente consistente con su posición, Berkeley podría haber mantenido una posición nominalista e instrumentalista con respecto a las matemáticas sin, por ello, negarles a los matemáticos la posibilidad de idear y de estudiar sistemas simbólicos. Él podría haber considerado el trabajo de los matemáticos, no como un estudio de las propiedades del mundo, sino de las propiedades y de las relaciones entre símbolos. Como veremos, su tendencia es la de seguir este camino. En los mismos Principios (PCH I, 122) expresa una opinión similar a ésta. Once años después de la publicación de los Principios, en 1721, Berkeley publica su tratado De Motu, en el que presenta una versión instrumentalista de términos claves de la física newtoniana de su tiempo:

Fuerza, gravedad, atracción y términos de este tipo son útiles para los razonamientos y cálculos acerca del movimiento y de los cuerpos en movimiento, pero no para entender la naturaleza simple del movimiento mismo o para indicar otras tantas cualidades distintas. (De Motu 17)

Y, en la siguiente sección, al hablar de la composición y de la resolución de fuerzas por medio del paralelogramo, nos dice:

Cumplen con el propósito de la ciencia y del cálculo mecánicos; pero que sirvan para el cálculo y las demostraciones matemáticas es una cosa, otra es presentar la naturaleza de las cosas. (De Motu 18) 
En el Alciphron (1732), ${ }^{5}$ Berkeley sostuvo la misma tesis nominalista $e$ instrumentalista acerca de las matemáticas:

Eufranor. Pero, aun cuando parece que ni tú ni yo podemos formar ideas simples de número, sin embargo podemos hacer un uso muy adecuado y significativo de las cifras. Éstas nos dirigen en la disposición y manejo de nuestros asuntos y son de un uso tan necesario que no sabríamos cómo arreglárnoslas sin ellas. (Alcphr. VII, 5)

Por tanto, la tesis de Berkeley es una que priva a los enunciados de las matemáticas - y, como lo señalé, también a algunos enunciados de la física- de su carácter descriptivo, que era la forma normal de considerarlos. Ahora bien, Berkeley niega no tan sólo que los enunciados matemáticos sean descriptivos de nuestra realidad perceptual, sino también rechaza la existencia de un reino platónico o de cualquier otra cosa que aquéllos puedan describir. De esta manera, el estudio de las matemáticas resulta ser uno puramente de símbolos y éstos adquieren su sentido por el uso práctico que se haga de ellos.

Pero, entonces, ¿c6mo es posible que esos enunciados no descriptivos resulten ser útiles? Es verdad que no sirven para descubrirnos nuevas verdades acerca del mundo puesto que carecen, en palabras de Berkeley, de la propiedad de establecer comparaciones entre ideas. Pero, conforme a la última cita del Alciphron, Berkeley ve tales enunciados o, para ser más exactos, las cifras mismas, como artificios o instrumentos que nos dirigen en la disposición y manejo de nuestros asuntos...

Antes de llegar a ver la manera como puedan "dirigirnos", quiero formular algunas consideraciones sobre lo que hasta aquí he presentado como las tesis de Berkeley sobre la aritmética.

${ }^{5}$ Cfr. [8] en la Bibliografía. 
Recordemos algunos de los pasajes antes citados en los que Berkeley subraya que no hay idea alguna relacionada con las expresiones numéricas. Un pasaje en el que es completamente explícito acerca de esto es el siguiente:

Éstas [la aritmética y el álgebra] son ciencias puramente Verbales y completamente inútiles a no ser para la Práctica de las Sociedades de los Hombres. No hay en ellas ningún conocimiento especulativo, ninguna comparación de Ideas. (CF 768)

Por tanto, como Berkeley considera los enunciados matemáticos, es posible concluir que no amplían nuestro conocimiento del mundo. Si añadimos los enunciados matemáticos al conjunto de nuestros enunciados factuales, no se obtendrá ninguna ampliación de nuestro conocimiento. En nuestros días, Hartry Field, según lo sugerí, ha presentado una posición similar a la de Berkeley, como una en la que la adición de nuestro sistema de enunciados matemáticos al de enunciados factuales $(M+F$, digamos), proporcionará, como resultado, una extensión conservadora de $F-$ del sistema de enunciados factuales-, en el sentido de que si es posible, de $M+F$, derivar un teorema, $T$ (que dependa esencialmente de algún(os) axioma(s) de $M$ ), entonces $T$ ya es un teorema de $F$ por sí solo. Ahora bien, obtener $T$ de $F$ por sí solo podría ser una labor ardua y difícil; la adición de $M$ a $F$ sólo simplifica la tarea de derivar teoremas, sin añadir otros nuevos.

Que podemos leer la posición de Berkeley conforme a la propuesta que acabo de delinear, se ve por el siguiente pasaje:

Ciertamente no es imposible que un hombre pueda llegar al conocimiento de toda verdad real tanto con como sin Signos si él tuviese una Memoria y una imaginación sumamente fuertes y capaces, por tanto el razonamiento y la ciencia no deben depender completamente de las Palabras o de los Nombres. (CF 883) 
Ésta es, ciertamente, una tesis extrema e inadmisible, que Berkeley felizmente modificó más tarde, como lo muestra el pasaje del Alciphron anterior. Pero cualquiera que fuese la tesis que Berkeley sostuviese, esta extrema o la otra más relajada, él fue consistente al considerar que los símbolos matemáticos tenían un carácter puramente intrumental, no "especulativo".

Por tanto, de acuerdo con Berkeley, el estudio del matemático es sólo de simbolismos, de lo que consisten las expresiones aritméticas o algebráicas sin que se adquiera, por su medio, ningún conocimiento, el que sea, de cualquier realidad extra simbólica quc uno quisiera considerar. Berkeley establece una relación entre la aritmética y el álgebra de tal naturaleza que la última la considera como el estudio de signos de signos; por tanto, se debe estudiar primero la aritmética:

Las letras o notaciones algebráicas son denominaciones de Denominaciones, por lo tanto tratar la Aritmética antes que el Álgebra. (CF 758)

En ningún caso, sea en la aritmética o en el álgebra, el estudio rebasa el nivel lingüístico. De manera contemporánea se podría decir que Berkeley restringe a los matemáticos al estudio de sistemas simbólicos no interpretados.

\subsection{Las tesis de Berkeley sobre la utilidad de la aritmética}

Pasemos ahora a la versión que dio Berkeley de la utilidad de los enunciados matemáticos. Uno de los pasajes más explícitos sobre este tema, en los $P C$, es el siguiente:

Estoy mejor informado y sabré más si me dicen que hay 10000 hombres que si me los muestran todos alineados. Estaré en mejor posición de juzgar el Negocio que quieres que haga cuando me digas cuánto es (esto es el nombre de) el dinero que yace sobre la Mesa que si me lo ofreces y me lo muestras sin nombrarlo. En breve yo no considero la Idea el aspecto sino los nombres. de esto puede surgir la Naturaleza de los Números. (CF 761) 
Puesto que Berkeley nos dice que está mejor informado al tener el número (la cifra) que cuando sólo tiene un conjunto de objetos ante él, podemos preguntar por cuál sea la cantidad extra de información que obtiene de esta manera.

Tomando en cuenta todo lo que ahora sabemos de las tesis de Berkeley, podemos leer el pasaje anterior como si nos dijera que por saber el número de cosas (hombres, dinero, etc.) que hay en algún momento, lugar, etc. dado, no llegamos a saber algo nuevo acerca del mundo. Lo que Berkeley diŕa es que el nombre (la cifra) aclara nuestras ideas acerca de cuánto hay de algo (más que..., lo mismo que..., menos que...). Pero entonces, saber esto, es saber algo acerca del mundo. Si nuestras ideas no fuesen claras acerca de algo, no tendríamos manera de decidir qué comportamiento adoptar. $\mathrm{Si}$, de alguna forma, llegamos a saber el número de... sabríamos si sería mejor correr o pelear, comprar o maldecir, etc.

La réplica de Berkeley creo que sería que ciertamente llegamos a saber algo de la manera como lo he indicado, pero esto no sería saber algo significativo acerca del mundo. Sólo llegamos a tener un conccimiento de cuántos hay de algo; esto sería una especie de conocimiento relacional, no entre ideas no lingüísticas que nos mostrase propiedades desconocidas de las mismas (gracias a esta relación), sino entre ideas y lenguaje (ideas de signos lingüísticos) que nos permite determinar la cardinalidad del conjunto de objetos ante nosotros, pero no nos dice qué sean los objetos, no nos dice nada acerca de la naturaleza intrínseca de las cosas. Lo que tendríamos sería sólo conocimiento práctico, no especulativo. Ésta es su idea de la aritmética y del álgebra como instrumentos útiles. Pero aún es importante insistir en que el considerar estos estudios de la manera como él lo hizo, no les permite ampliar su dominio y proporcionarnos nuevas herramientas útiles para enfrentarnos mejor a nuestros problemas prácticos.

Presento, finalmente, un pasaje del Alciphron en el que se 
resume la posición de Berkeley sobre el asunto que aquí nos ocupa:

quien entiende la notación de los números, por su medio es capaz de expresar, de manera breve y distinta, toda la diversidad y grados de los números y realizar con facilidad y eficiencia diversas operaciones matemáticas con ayuda de reglas generales. En tanto que, en la vida humana, el uso de todas esas operaciones es muy evidente, así no lo es menos que realizarlas depende de lo adecuado de la notación. Si suponemos a la ruda humanidad sin uso del lenguaje, puede presumirse que ignorase la aritmética. Pero el uso de los nombres cuya repetición en cierto orden podría expresar grados sin fin de números, sería el primer paso hacia esa ciencia. El siguiente sería idear marcas adecuadas, de una naturaleza permanente y visible, cuyo tipo y orden deben escogerse con juicio y acomodarse a los nombres. Marcas o notación que, en la medida en que fuera adecuada y regular facilitaría la invención y aplicación de reglas generales para ayudar a la mente a razonar y a juzgar, al extender, registrar y comunicar su conocimiento acerca de números, en cuya teoría y operaciones la mente se ocupa de manera inmediata acerca de los signos o notas, por los que la dirigen a actuar sobre las cosas, o número en concreto (como lo llaman los lógicos)... (Alcphr. VlI, 12)

\subsubsection{La propuesta relacional de Berkeley sobre la aritmética}

Lo que más subraya Berkeley es que los enunciados de la aritmética no sirven para comparar ideas; tales enunciados no amplían nuestro conocimiento del mundo, sino sólo establecen relaciones entre signos. Es, dentro de esta posición, donde encontramos la propuesta más interesante y promisoria de Berkeley: los números no son sino relaciones 0 , sería mejor decir que los números son elementos o puntos nodales en una estructura relacional. Esta tesis acerca de los números proviene de considerar diversos aspectos de la posición de Berkeley: (a) la aritmética es un estudio puramente nominalista; por tanto, (b) no es descriptiva de este mundo ni de ningún otro; pero (c) la aritmética es útil como una guía de nuestra experiencia. Si reuni- 
mos las propuestas (b) y (c) podemos concluir que la aritmética, para Berkeley, es un instrumento útil en nuestro proceso de adquirir nuevos conocimientos y de ampliar los que ya tenemos. Pero, ¿cómo es esto posible?

Para responder la pregunta anterior de Berkeley, me apoyaré en sus palabras del Alciphron: "el uso de los nombres cuya repetición en cierto orden podría expresar grados sin fin de números, sería el primer paso hacia esa ciencia. El siguiente sería idear marcas adecuadas, de una naturaleza permanente y visible, cuyo tipo y orden deben escogerse con juicio y acomodarse a los nombres. Marcas o notación que, en la medida que fuera adecuada y regular facilitaría la invención y aplicación de reglas generales para ayudar a la mente a razonar y a juzgar". Esta generación ordenada de los números (= palabras o nombres en la versión berkeleyana; esto, conforme a la versión de Benacerraf, sería contar de manera intransitiva) ${ }^{6}$ y la subsecuente generación de marcas para referirse a los primeros objetos intento reconstruirla en las siguientes líneas.

6 Op. cui., p. 274. En ese lugar Benacerraf nos dice: "nosotros normalmente aprendemos unos cuantos de los primeros números en conexión con conjuntos que tienen ese número de elementos —esto es, en términos de contar transitivo (aprendiendo, así, a usar los números) y luego aprendemos cómo generar 'el resto' de los números. De hecho, 'el resto' queda siempre como un asunto relativamente vago. La mayoría de nosotros simplemente aprendemos que nunca nos quedaremos cortos, que nuestra notación se extenderá tanto como jamás necesitemos contar. Aprender estas palabras y cómo repetirlas es aprender el contar intransitivo. [El subrayado me pertenece.] Aprender su uso como medida de conjuntos es aprender el contar transitivo. Sea que aprendamos un tipo de contar antes que el otro no importa con respecto a los números iniciales. Lo que es cierto y sí importa es que tendremos que aprender algún procedimiento recursivo para generar la notación en el orden adecuado antes de que hayamos aprendido a contar transitivamente pues, hacer esto es, de manera directa o indirecta, correlacionar los elementos de la serie de números con los miembros del conjunto que estamos contando." ([2], pp. 274-275.)

Me parece, de manera clara, que Berkeley no tendría ningún reparo en aceptar lo anterior, pues lo mismo se desprende de la cita de él que aquí comento. 
Presento, primero, una lista de signos, $(\operatorname{Pr})$, y un par de definiciones $D f_{1}$ y $D f_{2}$ :

$$
*, a, g, m, k, r, b, c, f, e ;
$$

éstos los tomo como mis signos básicos o primitivos. Las definiciones son:

$\left(D f_{1}\right)$

Expresiones:

(i) Cualquiera de los signos de $(P r)$ es una expresion.

(ii) Si $x$ es una expresión y $w$ es un signo de $(P r)$,

$$
((x) w)
$$

es también una expresión.

(iii) Las expresiones son sólo las concatenaciones de símbolos que puedan obtenerse por medio de un número finito de aplicaciones de las cláusulas (i) y/o (ii) anteriores.

$\left(D f_{2}\right)$

\section{Sucesor:}

Sea $x$ cualquier expresión y

(i) sea $w$ un signo de $(\operatorname{Pr})$ distinto de $e$, entonces:

$$
((x) w)^{\prime}=\left((x) w^{\prime}\right),
$$

donde $w^{\prime}$ es el signo inmediatamente a la derecha de $w$ en la lista $(P r)$.

$\mathrm{O}$ bien,

(ii) si $w=e$, entonces:

$$
\begin{aligned}
((x) w)^{\prime} & =((x) e)^{\prime} \\
& =\left((x)^{\prime} *\right),
\end{aligned}
$$

donde hemos de considerar dos casos: 
(a) Si $x=e$, entonces:

$$
\begin{aligned}
(x)^{\prime} & =(e)^{\prime} \\
& =((a) *) .
\end{aligned}
$$

(b) Si $x \neq e$, entonces procédase como en (i).

Puesto que, conforme a la $D f_{1}$, toda expresión es una concatenación finita de paréntesis y de signos de (Pr), entonces $x$ es finita y en un número finito de pasos concluiremos las aplicaciones de las cláusalas (i) y/o (ii) de la $D f_{2}$.

Por último, para poner a funcionar la maquinaria formal, tomo como axioma o punto de partida, para construir un sistema ordenado de expresiones, la siguiente expresión, $(A)$, que, como lo puede verificar el lector, cumple con las especificaciones de la $D f_{1}$ :

$$
((*) *) \text {. }
$$

Partiendo de $(A)$ y mediante sucesivas aplicaciones de la función de la $D f_{2}$, presento de inmediato una porción mínima de las expresiones que pueden obtenerse mediante el uso de estas herramientas:

$$
\begin{gathered}
((*) *),((*) a),((*) g), \ldots,((*) c),((*) f),((*) e), \\
((a) *),((a) a),((a) g), \ldots,((a) c),((a) f),((a) e) \\
\ldots \\
((e) *),((e) a),((e) g), \ldots,((e) c),((e) f),((e) e) \\
(((a) *) *),(((a) *) a), \ldots,(((a) *) f),(((a) *) e) \\
\ldots \\
((((((e) e) e) e) f) *), \ldots,((((((e) e) e) e) f) e) \\
((((((e) e) e) e) e) *), \ldots,((((((e) e) e) e) e) e) \\
(((((((a) *) *) *) *) *) *), \ldots
\end{gathered}
$$

El proceso puede continuarse indefinidamente y, de esta manera, obtenemos una lista ordenada de expresiones cuyo significado está dado por el lugar que ocupan en esta ordenación. Puesto que el único operador empleado para construir la lista 
anterior es la función de la $D f_{2}$, podemos estar seguros de que no habrá dos lugares diferentes que contengan la misma expresión; esto es, lo que puede mostrarse es que la función de la $D f_{2}$ es $1-1$. Por otra parte, también es posible obtener el siguiente resultado: dada una expresión cualquiera, que sea posible obtener por el procedimiento anterior -tomando $(A)$ como punto de partida-, podemos determinar, con precisión total, la distancia a la que se encuentra del punto de partida — de $(A)$ - en el sentido de saber cuáles son todas las expresiones que vienen antes que ella y su orden. Esto nos permitiría, igualmente, hacer comparaciones entre las posiciones relativas de dos o más expresiones diferentes, etc.

La notación que usé puede simplificarse considerablemente -y sin muchos problemas - eliminando los paréntesis, por ejemplo. Sin embargo, creo que puedo ofrecer algo más simple, algo con lo que mi lector ha estado familiarizado desde hace mucho. Lo que me ha llevado tanto tiempo construir, no es otra cosa que nuestro bien conocido sistema de números naturales, que tiene las propiedades que arriba he enumerado y otras más que el lector conoce muy bien.

La razón de lo anterior es que considero que ésta es una forma de poner de manifiesto lo que está implícito en los pensamientos de Berkeley acerca de la aritmética, la que toma como un estudio nominalista (instrumentalista, utilitario, relacional). En la construcción anterior, los signos que configuran las expresiones tienen significado sólo en tanto que ocupan un lugar determinado dentro de la ordenación generada por la función de la $D f_{2}$. Esto ya lo he señalado. Pero, además, como me lo sugirió R. Orayen, ${ }^{7}$ las cifras pueden verse como símbolos incompletos o como expresiones sincategoremáticas (en un sentido amplio del término) que obtienen su significado total en contextos de

7 Fue como una respuesta a una sugerencia de R. Orayen que escribí esta parte de mi propuesta interpretativa de las tesis de Berkeley. Mis dos definiciones las discutí con R. Morado. A ambos les agradezco su tiempo y sus lúcidas observaciones sobre estos temas. 
uso. La ordenación que se genera en el sistema que he presentado - la decimal — sirve como un patrón - como una regla para medir la cardinalidad de conjuntos de objetos: tales comparaciones de tamaño pueden llevarse a cabo entre conjuntos de objetos por medio de la sucesión de las expresiones generadas. Por otra parte, nótese que en todo el análisis anterior no se ha hecho ninguna mención explícita de números o de cualesquiera otras entidades numéricas, diferentes de las expresiones mismas. Por tanto, lo que aquí he presentado creo que puede verse como un ejemplo de sistema aritmético berkeleyano.

Por último, para eliminar del lector las mínimas dudas que aún pueda tener acerca de cómo tomar mi formulación del sistema de expresiones, le pregunto si no es verdad que, cuando hablamos de números bastante grandes, la única cosa que tenemos y que podemos tener en mente es la notación que los expresa (esta propuesta ya la formularon con anterioridad, entre otros, Locke y Berkeley). Por ejemplo, sabemos que 55663857901 es el sucesor inmediato de 55663857900 y esto sólo debido al sistema de símbolos y no porque tengamos una intuición clara y precisa de los números mismos (como algo diferente del conjunto de símbolos) que expresa cada una de las cifras anteriores. Es debido a la notación posicional que adquieren su significado las expresiones anteriores, y no debido a la "entidad" —en caso de que la hubiere_ a la que supuestamente se refieren. En estos casos podemos explicar mejor la significatividad de las cifras mediante una posición berkeleyana nominalista, que mediante una platónica realista.

\section{La propuesta de Benacerraf}

Lo que aquí me resta por hacer es mostrar que Paul Benacerraf, en su importante artículo de 1965, al que ya aludí, nos presenta y aclara, en terminología contemporánea, la propuesta que Berkeley formulara hace ya más de 200 años. Ciertamente, Benacerraf introduce observaciones y precisiones ajenas a los 
conocimientos de los autores de la época de Berkeley. Sin embargo, me parece que el núcleo nominalista, relacional y pragmático, es esencialmente el mismo en ambos autores.

En primer lugar, la posición nominalista Benacerraf la presenta con claridad en su artículo. Pero aquí lo interesante es la manera como declara que es la estructura, y sólo ella, la que confiere significado a las expresiones numéricas:

Para los propósitos aritméticos no tienen consecuencia alguna, cualquiera que ésta sea, las propiedades de los números que no surjan de las relaciones que mantienen entre sí por virtud de estar ordenados en una progresión. Y sólo serían estas propiedades las que distinguirían un número como uno u otro objeto cualquiera. (Op. cit., p. 291)

Que un sistema de objetos exhiba la estructura de los enteros implica que los elementos de ese sistema tienen algunas propiedades que no dependen de la estructura. Debe de ser posible individualizar esos objetos de manera independiente al papel que desempeñan en esa estructura. Pero esto es precisamente lo que no puede hacerse con los números. Ser el número 3 no es, ni más ni menos, que estar precedido por 2,1 y posiblemente 0 . (lbid.)

Por el momento no deseo detenerme en presentar más citas del texto de Benacerraf; lo que ahora haré será formular unos breves comentarios generales sobre su propuesta.

En primer lugar, algo que es muy importante tener en cuenta es que, conforme a Benacerraf, la estructura numérica no hay que verla como una estructura conjuntística. Los números no son objetos, sino meros puntos nodales en una estructura generada de manera recursiva. Que puedan configurarse conjuntos que se comportan a la manera como se comportan tales puntos nodales es algo que no debe hacernos pensar que los números son conjuntos. Aquí me interesa volver a repetir una propuesta de Benacerraf: ser el número 1 es sólo una propiedad estructural del primer punto nodal en la estructura recursiva. 
Los números, así tomados, no tienen unidades; lo que puede "tener" unidades son los conjuntos de unidades; dado que los números no son conjuntos, no puede haber una relación de pertenencia de algo, $x$, con respecto a ellos. Considero que ésta es una —o quizás $l a$ - propuesta realmente importante en la tesis de Benacerraf, a la que vuelve más adelante en su ensayo y nos dice:

Al contar, no correlacionamos conjuntos con segmentos iniciales de números como entidades extralingüísticas, sino que correlacionamos conjuntos con segmentos iniciales de la sucesión de palabras numéricas. La idea central es que esta sucesión recursiva es una especie de regla [yard-stick] que usamos para medir conjuntos. (Op. cit., p. 293)

Aquí podemos ver lo absurdo de preguntar, por ejemplo, por cuántos centímetros hay en el centímetro 3 de la regla. En una regla cada centímetro es distinto a cualquier otro sólo por la posición que cada uno de ellos ocupa en la misma. La función de nuestra estructura numérica recursiva es la de permitirnos "medir", de manera no problemática, cardinalidades de conjuntos. Estas medidas nos permitirán llevar a cabo comparaciones de tamaño de conjuntos, donde la regla misma cumple sólo con la tarea de intermediario en el proceso de comparación.

Debido al proceso de generación de nuestras unidades numéricas, sabemos qué unidades aparecen antes de cuáles otras. A las que aparecen primero las denominamos menores que aquellas que aparecen después y así, si al agotar un conjunto de objetos, $C$, recorremos nuestra regla hasta el lugar $m$ y al agotar otro conjunto de objetos, $D$, nuestro recorrido alcanza hasta el lugar $n$, y si $m$ es menor que $n$, entonces $\kappa(C)<\kappa(D)$-donde “ $\kappa(x)$ " se lee "el cardinal de $x$ ". Una vez que cumple con su cometido la regla desaparece y no deja, como residuo, alguna entidad numérica relacionada con los objetos que forman los conjuntos enumerados. 


\section{Conclusión}

Tras este breve recorrido en el que presenté las tesis nominalistas estructurales de Berkeley y de Benacerraf, pueden extraerse las siguientes conclusiones: las propuestas de Berkeley de los años 1707-1731 coinciden de manera esencial con las de Benacerraf de 1965. En este sentido podemos adjudicarle a Berkeley el papel de precursor desconocido de algunas esenciales propuestas nominalistas contemporáneas.

Por otra parte, con respecto a la evaluación de la doctrina, la misma procede de manera clara y poco misteriosa, a diferencia de aquellas en las que se postulan entidades curiosas como los números. Mi impresión general siempre ha sido que tal postulación viola creencias naturales, profundamente arraigadas en nuestra visión de la realidad. Por esto, me parece fructífero encontrar propuestas explicativas, de los diferentes sectores de la realidad, donde se muestre que tales postulaciones de entidades abstractas son innecesarias. ${ }^{8}$

Aquí es preciso aclarar algo que me pesa profundamente en la conciencia. A pesar de la simplificación que ontologicamente representa el eliminar los números como entidades extralingüísticas de algún tipo, sin embargo el nominalista no se ve libre de problemas de ontología. Ciertamente creo que necesita algún tipo de andamiaje abstracto para lograr lo que desea: formular una (única) teoría matemática que compartan todos los matemáticos. Desea que sus propuestas sean objetivas y generales y, para esto último, surge la necesidad de hablar de una estructura común a todas las sucesiones tales o cuales y esta estructura común tiene las características de un universal, de una entidad abstracta.

Se podría alegar que hay cierto tipo de abstracciones que son más fáciles de explicar que otras; que podríamos dar una

8 Para ver alguna comparación de algunas propuestas realistas (formuladas en la época de Berkeley) con el nominalismo berkeleyano, cfr. mi [11]. Este mismo escrito lo añadí como Apéndice al capítulo 2 de mi libro sobre Berkeley, [12] en la Bibliografía. 
versión explicativa funcional de lo que sea una estructura numérica, que no nos llevara a proponer entidades abstractas, pero no me convence ninguna propuesta en este sentido. El texto de Keith Hossack que se publica en este mismo volumen pretende encontrar una salida nominalista al problema, pero no es claro que pueda evitar el cargo de que el ver similitudes entre estructuras es ya "ver" algún tipo de universalidad del tipo que no se desea.

Mi conclusión, nuevamente, puedo formularla en términos similares a los de los dos párrafos atrás: "me parece fructífero encontrar propuestas explicativas, de los diferentes sectores de la realidad, donde se muestra que tales postulaciones de entidades asbtractas son innecesarias", y lo que podría añadir a esta observación es que la propuesta formalista es, en un sentido claro, al menos más económica ontológicamente que la propuesta realista y, en este sentido, la encuentro preferible a aquélla, aun cuando no sea perfecta.

\section{BIBLIOGRAFÍA}

[1] Ball, W.W.R., A Short Account of the History of Mathematics (1889), 2a. ed., Dover Publications, Inc., Nueva York, 1966.

[2] Benacerraf, P., "What numbers could not be", Philosophical Review, no. 74, 1965, pp. 47-73; recogido en P. Benacerraf y H. Putnam (comps.), Philosophy of Mathematics, 2a. ed., Cambridge University Press, Nueva York, 1983, pp. 272-294. Las citas las hago de esta última edición.

[3] Berkeley, G., Philosophical Commentaries, más conocido como el Libro del lugar común (escrito en: 1707-1708). Una editio diplomata transcrita y editada con una introducción y notas de A.A. Luce, Thomas Nelson and Sons, London, 1944. Hay traducción al español: Comentarios filosóficos, Introducción manuscrita a "Los principios del conocimiento humano", Correspondencia con Johnson, introducción, traducción y notas de José Antonio Robles, Instituto de Investigaciones Filosóficas, UNAM, México, 1989. 
[4] __ The Works of George Berkeley Bishop of Cloyne, vols. I-IX, editado por A.A. Luce y T.E. Jessop, Thomas Nelson and Sons, Ltd., Londres, 1948-1957.

[5]_, Philosophical Commentaries (redacción 1707-1708), Introducción (pp. 3-6) y notas (pp. 107-139) de A.A. Luce, y texto (pp. 9-104), en [4] vol. II.

[6] _ A Treatise Concerning the Principles of Human Knowledge, Wherein the chief causes of error and difficulty in the Sciences, with the grounds of Scepticism, Atheism, and Irreligion are inquired into (1710). Introducción (pp. 3-7) de T.E. Jessop, texto (pp. 9-113), en [4] vol. II.

[7]—_ De Motu, sive de motus principio \& natura et de Causa comunicationis motrum (1721). Introducción (pp. 3-7) y traducción inglesa Of Motion, or the principle and nature of motion and the cause of the communication of motions (pp. 31-52) de T.E. Jessop, texto (pp. 9-30), en [4] vol. IV. Hay traducción al español: Sobre el movimiento o Sobre el Principio y la Naturaleza del Movimiento y Sobre la Causa de la Transmisión de los Movimientos (1721). Traducido del latín por Roberto Torretti, Diálogos, Revista del Departamento de Filosofía, Universidad de Puerto Rico, vol. XIV, no. 34, noviembre 1979, pp. 119-141.

[8]___, "Alciphron or the Minute Philosopher" in seven dialogues; containing "An Apology" for the "Christian Religion", against those who are called "Free-Thinkers" (1931). Hay traducción al español: Alcifrón o el filósofo minucioso, de Pablo García Castillo, introducción y notas de Cirilo Flórez Miguel, Ediciones Paulinas, Madrid, 1978 (Cuadernos Salmantinos de Filosofía).

[9]__, Letters (1709-1752), prefacio, introducción y notas de A.A. Luce, en [4] vol. VIII.

[10] Field, H., Science Without Numbers: a Defense of Nominalism, Basil Blackwell, Oxford, 1980.

[11] Robles, J.A., "Matemática hasta el siglo XVIII. ¿Qué son los números?", Diánoia, 1982, pp. 155-171.

[12]—_ Las ideas matemáticas de George Berkeley (en prensa). 
In this paper I point out that besides having made a proposal —with respect to arithmetic (and algebra) - which makes Berkeley an antecesor of Hartry Field — something which I do not elaborate more in this paper-, he also puts forth a nominalistic view which, in substantial points, is closely related to Benacerraf's in his "What Numbers Could Not Be". What I hold is that Berkeley's view, two hundred years before Benacerraf's, fullfils the latter's claim for numerical expressions to be meaningful and to make them useful in human practices.

I interpret some proposals by Berkeley which would ground the construction of a mathematical structure in which no use is made of sets or any type of entities - besides the structure itself, of which I give an example-, to give meaning to the expressions which constitute it. Such expressions get their meaning from the proposition they have in the said structure. I take it that a nominalistic position, at least at the level of elementary mathematics, avoids many theoretical problems which arise if we adopt a less econornical ontological position. 